PROCEEDINGS OF THE

AMERICAN MATHEMATICAL SOCIETY

Volume 139, Number 12, December 2011, Pages 4217-4223

S 0002-9939(2011)10935-3

Article electronically published on April 26, 2011

\title{
A CHARACTERISATION OF ANTI-LÖWNER FUNCTIONS
}

\author{
KOENRAAD M. R. AUDENAERT
}

(Communicated by Marius Junge)

\begin{abstract}
According to a celebrated result by Löwner, a real-valued function $f$ is operator monotone if and only if its Löwner matrix, which is the matrix of divided differences $L_{f}=\left(\frac{f\left(x_{i}\right)-f\left(x_{j}\right)}{x_{i}-x_{j}}\right)_{i, j=1}^{N}$, is positive semidefinite for every integer $N>0$ and any choice of $x_{1}, x_{2}, \ldots, x_{N}$. In this paper we answer a question of R. Bhatia, who asked for a characterisation of real-valued functions $g$ defined on $(0,+\infty)$ for which the matrix of divided sums $K_{g}=$ $\left(\frac{g\left(x_{i}\right)+g\left(x_{j}\right)}{x_{i}+x_{j}}\right)_{i, j=1}^{N}$, which we call its anti-Löwner matrix, is positive semidefinite for every integer $N>0$ and any choice of $x_{1}, x_{2}, \ldots, x_{N} \in(0,+\infty)$. Such functions, which we call anti-Löwner functions, have applications in the theory of Lyapunov-type equations.
\end{abstract}

\section{INTRODUCTION}

A real-valued function defined on an interval $(a, b)$ is called matrix monotone of order $N$ if for any pair $A, B$ of $N \times N$ Hermitian matrices with spectrum in $(a, b)$ the implication $A \leq B \Longrightarrow f(A) \leq f(B)$ holds; i.e. $f$ preserves the positive semidefinite ordering. A function is called operator monotone if it is matrix monotone of every order.

One of the central objects in the theory of matrix monotone functions is the so-called Löwner matrix. Given any integer $N>1$ and any set of $N$ finite, distinct real numbers $x_{i}$ in $(a, b)$, one constructs a Löwner matrix of $f$ as the $N \times N$ matrix $L_{f}$ of divided differences

$$
L_{f}:=\left(\frac{f\left(x_{i}\right)-f\left(x_{j}\right)}{x_{i}-x_{j}}\right)_{i, j=1}^{N} .
$$

For the diagonal elements, $i=j$, a limit has to be taken so that the diagonal elements are given by the first derivatives $f^{\prime}\left(x_{i}\right)$. (A necessary condition for $f$ being matrix monotone of order at least 2 is that it should be continuous, in fact even continuously differentiable ([3], p. 79); hence its first derivative should exist. For $N=1$, this is strictly speaking not needed, but matrix monotonicity then reduces to ordinary monotonicity anyway.) According to a celebrated result by Löwner, $f$ is a matrix monotone function on $(a, b)$ of order $N$ if and only if any $N \times N$ Löwner matrix $L_{f}$ is positive semidefinite, for any choice of $x_{i}$ in $(a, b)$. For

Received by the editors October 20, 2010.

2010 Mathematics Subject Classification. Primary 15A60.

Key words and phrases. Matrix monotone functions, Löwner matrices, Lyapunov equation.

(C)2011 American Mathematical Society Reverts to public domain 28 years from publication 
a thorough introduction to matrix monotone functions we refer to the monograph [3] and to [1] for a more concise introduction.

In ([2, p. 195) R. Bhatia raised the question of whether there is a good characterisation of real-valued functions $g(x)$ defined on $(a, b)$, with $a \geq 0$, for which every matrix of the form

$$
K_{g}:=\left(\frac{g\left(x_{i}\right)+g\left(x_{j}\right)}{x_{i}+x_{j}}\right)_{i, j=1}^{N}
$$

is positive semidefinite, with $x_{i}$ distinct real numbers in $(a, b)$. That is, $K_{g}$ is akin to a Löwner matrix but has the minus signs replaced by plus signs. In this paper, we will call these matrices anti-Löwner matrices, and functions for which all $N \times N$ anti-Löwner matrices are positive semidefinite will be called anti-Löwner functions of order $N$. Likewise, we call functions anti-Löwner functions if they satisfy this positivity criterion for all values of $N$.

It goes without saying that to be anti-Löwner $g$ must first of all be non-negative, as can be seen from the trivial case $N=1$. For $N=1$ this is already the complete answer; to avoid trivialities we will henceforth assume $N \geq 2$. It is also straightforward to show that for $N \geq 2, g$ must be continuous, similar to matrix monotone functions of order $N \geq 2$; see Proposition 1 below.

It has been known for some time that every non-negative operator monotone function on $(0,+\infty)$ is an anti-Löwner function, and so is every non-negative operator monotone decreasing function [5. This easily follows (see, e.g., Theorem 1 in [5]) from exploiting the well-known integral representation [1]

$$
f(x)=\alpha+\beta x+\int_{0}^{\infty} \frac{x}{t+x} d \mu(t)
$$

for non-negative operator monotone functions on $(0,+\infty)$, with $\alpha, \beta \geq 0^{1}$ and $\mu$ a positive Borel measure on $(0,+\infty)$ such that the given integral converges. The statement for non-negative operator monotone decreasing functions follows easily from this by noting that the function $f(x)$ is anti-Löwner if and only if $1 / f(x)$ is anti-Löwner too.

\section{MAIN RESULTS}

In this paper, we obtain a complete answer to Bhatia's question:

Theorem 2.1. Let $g$ be a real-valued function $g$, defined and finite on $(a, b)$, with $0 \leq a<b$. Let $N$ be any integer, at least 2 . If $g$ is an anti-Löwner function of order $N$ on $(a, b)$ then $x \mapsto g(\sqrt{x}) \sqrt{x}$ is a non-negative matrix monotone function of order $N$ on $\left(a^{2}, b^{2}\right)$ and $x \mapsto g(\sqrt{x}) / \sqrt{x}$ is a non-negative matrix monotone decreasing function of order $N$ on $\left(a^{2}, b^{2}\right)$.

Theorem 2.1 has applications in the study of Lyapunov-type equations and also answers a question by Kwong [5, who studied conditions on the function $g$ such that the solution $X$ of equation $A X+X A=g(A) B+B g(A)$ is positive definite for all positive definite $A$ and $B$. Kwong pointed out in 4 that it suffices to consider matrices $A$ that are diagonal, with $B$ equal to the all-ones matrix $\left(B_{i j}=1\right)$. In that case the solution of the equation reduces to $X$ being an anti-Löwner matrix

\footnotetext{
${ }^{1}$ Note that for negative $\alpha, f$ is also operator monotone, but positivity of $f$ requires $\alpha \geq 0$.
} 
with the $x_{i}$ equal to the diagonal elements of $A$. Thus, our Theorem 2.1 also yields the answer to Kwong's question.

As a direct corollary of Theorem 2.1, we immediately get an integral representation for anti-Löwner functions $g(x)$ (of all orders) on $(0,+\infty)$. From equation (1.1) we obtain $g(\sqrt{x})=\alpha / \sqrt{x}+\beta \sqrt{x}+\int_{0}^{\infty} \frac{\sqrt{x}}{t+x} d \mu(t)$. Hence

$$
g(x)=\frac{\alpha}{x}+\beta x+\int_{0}^{\infty} \frac{x}{t+x^{2}} d \mu(t),
$$

with $\alpha, \beta \geq 0$ and $\mu$ a positive Borel measure such that the integral exists.

Within this restricted setting, the sufficiency part of Theorem 2.1 is easy to prove, as it suffices to check each of the terms in the integral representation (2.1). To wit, one only needs to prove that the functions $g(x)=x$ and $g(x)=x /\left(t+x^{2}\right)$ (for $t \geq 0$ ) are anti-Löwner, as all other functions concerned are positive linear combinations of these extremal functions. This is trivial for $g(x)=x$, because then $K_{g}=(1)_{i, j}$, which is clearly positive semidefinite (and rank 1 ). Secondly, for $g(x)=x /\left(t+x^{2}\right)$, we have

$$
\begin{aligned}
K_{g} & =\left(\frac{x_{i} /\left(t+x_{i}^{2}\right)+x_{j} /\left(t+x_{j}^{2}\right)}{x_{i}+x_{j}}\right)_{i, j} \\
& =\left(\frac{x_{i}\left(t+x_{j}^{2}\right)+x_{j}\left(t+x_{i}^{2}\right)}{\left(t+x_{i}^{2}\right)\left(x_{i}+x_{j}\right)\left(t+x_{j}^{2}\right)}\right)_{i, j} \\
& =\left(\frac{t+x_{i} x_{j}}{\left(t+x_{i}^{2}\right)\left(t+x_{j}^{2}\right)}\right)_{i, j},
\end{aligned}
$$

which is congruent to the matrix $\left(t+x_{i} x_{j}\right)_{i, j}$ and therefore positive semidefinite as well (and in general rank 2).

The hard part is to prove necessity, i.e. that there are no other anti-Löwner functions than those with the given integral representation (2.1). Furthermore, there seems to be no obvious approach even to the sufficiency part in the more general setting of fixed $N$ where no integral representation is known. An important observation that shows the way out, however, is hidden in the very statement of Theorem 2.1, as it hints at a one-to-one correspondence between anti-Löwner functions and non-negative operator monotone functions. This is no coincidence, and our method of proof will exploit an even deeper correspondence between Löwner matrices and anti-Löwner matrices, which is made apparent in Theorem 2.2 below. This is good news, as there will be no need to develop from scratch a completely new theory in parallel with Löwner's.

Theorem 2.2. Let $N$ be any integer and $x_{1}, \ldots, x_{N}$ be a sequence of distinct positive real numbers contained in the interval $(a, b), 0 \leq a<b$. For any continuous real-valued function $g$ defined on $(a, b)$, let $L$ and $K$ be its Löwner and anti-Löwner matrix of order $N$ on the given points $x_{1}, \ldots, x_{N}$, respectively, and let $K_{i j}$ be the matrix

$$
K_{i j}=\left[\frac{g\left(x_{k}+i \varepsilon\right)+g\left(x_{l}+j \varepsilon\right)}{\left(x_{k}+i \varepsilon\right)+\left(x_{l}+j \varepsilon\right)}\right]_{k, l=1}^{N}
$$


and let $L_{i j}$ be the matrix

$$
L_{i j}=\left[\frac{g\left(x_{k}+i \varepsilon\right)-g\left(x_{l}+j \varepsilon\right)}{\left(x_{k}+i \varepsilon\right)-\left(x_{l}+j \varepsilon\right)}\right]_{k, l=1}^{N} .
$$

Then the following are equivalent:

(1) the $2 \times 2$ block matrix $\left(\begin{array}{ll}K_{00} & K_{01} \\ K_{10} & K_{11}\end{array}\right)$ is positive semidefinite,

(2) the $2 \times 2$ block matrix $\left(\begin{array}{cc}K_{00} & L_{01} \\ L_{10} & K_{11}\end{array}\right)$ is positive semidefinite.

In the remainder of this paper we present the proofs of these theorems.

\section{ProOfs}

We start with a simple, but nevertheless essential, proposition.

Proposition 1. Let $g$ be a positive real-valued function on $(a, b)$, with $0 \leq a<b$. If $g$ is an anti-Löwner function of order at least 2 , then $g$ is continuous.

Proof. This follows from consideration of the $2 \times 2$ anti-Löwner matrices in the points $x_{1}=x, x_{2}=x+\epsilon(0 \leq a<x<b)$ and letting $\epsilon$ tend to 0 . Positive semidefiniteness of the anti-Löwner matrix requires non-negativity of its determinant:

$$
g(x) g(x+\epsilon) / x(x+\epsilon)-(g(x)+g(x+\epsilon))^{2} /(2 x+\epsilon)^{2} \geq 0 .
$$

After some calculation, one finds that this requires $|(g(x+\epsilon)-g(x)) / \epsilon| \leq g(x) / x$ for all $\epsilon>0$, whence the derivative of $g$ should exist and be bounded on any bounded closed interval in $(a, b)$.

The main technical result on which our proof is based is the following proposition.

Proposition 2. Fix an integer $N$. Let $g=\left(g_{1}, \ldots, g_{N}\right)$ and $x=\left(x_{1}, \ldots, x_{N}\right)$ be positive vectors, where all $x_{i}$ are distinct, and let $s=\left(s_{1}, \ldots, s_{N}\right)$ be a real vector with $s_{i}= \pm 1$. Then the sign of $\operatorname{det} Z_{N}$, where

$$
Z_{N}=\left(\frac{s_{i} g_{i}+s_{j} g_{j}}{s_{i} x_{i}+s_{j} x_{j}}\right)_{i, j=1}^{N},
$$

is independent of the signs of the $s_{i}$ 's.

As an illustration of this proposition, we will prove the easiest non-trivial case $N=2$ (the case $N=1$ is trivial as $s_{1}$ cancels out entirely). The given determinant is

$$
\begin{aligned}
\operatorname{det} Z_{2} & =\operatorname{det}\left(\begin{array}{cc}
\frac{g_{1}}{x_{1}} & \frac{s_{1} g_{1}+s_{2} g_{2}}{s_{1} x_{1}+s_{2} x_{2}} \\
\frac{s_{1} g_{1}+s_{2} g_{2}}{s_{1} x_{1}+s_{2} x_{2}} & \frac{\underline{g}_{2}}{x_{2}}
\end{array}\right) \\
& =\frac{g_{1} g_{2}}{x_{1} x_{2}}-\frac{\left(s_{1} g_{1}+s_{2} g_{2}\right)^{2}}{\left(s_{1} x_{1}+s_{2} x_{2}\right)^{2}} \\
& =\frac{g_{1} g_{2}\left(s_{1}^{2} x_{1}^{2}+s_{2}^{2} x_{2}^{2}\right)-x_{1} x_{2}\left(s_{1}^{2} g_{1}^{2}+s_{2}^{2} g_{2}^{2}\right)}{x_{1} x_{2}\left(s_{1} x_{1}+s_{2} x_{2}\right)^{2}} \\
& =\frac{g_{1} g_{2}\left(x_{1}^{2}+x_{2}^{2}\right)-x_{1} x_{2}\left(g_{1}^{2}+g_{2}^{2}\right)}{x_{1} x_{2}\left(s_{1} x_{1}+s_{2} x_{2}\right)^{2}} .
\end{aligned}
$$


One sees that the numerator is independent of the signs of the $s_{i}$ 's, while the denominator is always positive. Hence, the sign of this determinant is independent of the signs of the $s_{i}$ 's.

For small values of $N$ one can easily verify that the determinant can always be written as a rational function where the numerator is a polynomial in which the $s_{i}$ 's only appear to even powers and where the denominator is always positive. This observation provided the inspiration for the following simple proof of Proposition 2 (for every value of $N$ ).

Proof of Proposition 2. Clearly, once we prove that the sign of $\operatorname{det} Z_{N} \operatorname{does}$ not change under a single sign change of $s_{i}$, the general statement of the proposition follows by changing the signs of the $s_{i}$ 's one by one. W.l.o.g. we consider sign changes of $s_{1}$ only.

The idea of the proof is to apply a partial Gaussian elimination on $Z_{N}$, only bringing its first column in upper-triangular form. For each $i>1$ we subtract $\frac{x_{1}}{g_{1}} \frac{s_{1} g_{1}+g_{i}}{s_{1} x_{1}+x_{i}}$ times row 1 from row $i$. As is well-known, this operation does not change the determinant. The resulting matrix is of the form

$$
Z_{N}^{\prime}=\left(\begin{array}{cc}
\frac{g_{1}}{x_{1}} & b \\
0 & X
\end{array}\right),
$$

where $b$ is the first row of $Z_{N}$ (except its element $\left.(1,1)\right)$ and $X$ is an $(N-1) \times(N-1)$ matrix with elements $(i, j>1)$ :

$$
\begin{aligned}
X_{i, j} & =\frac{g_{i}+g_{j}}{x_{i}+x_{j}}-\frac{x_{1}}{g_{1}} \frac{s_{1} g_{1}+g_{i}}{s_{1} x_{1}+x_{i}} \frac{s_{1} g_{1}+g_{j}}{s_{1} x_{1}+x_{j}} \\
& =\frac{g_{1}\left(g_{i}+g_{j}\right)\left(x_{1}^{2}+x_{i} x_{j}\right)-x_{1}\left(x_{i}+x_{j}\right)\left(g_{1}^{2}+g_{i} g_{j}\right)}{g_{1}\left(x_{i}+x_{j}\right)\left(s_{1} x_{1}+x_{i}\right)\left(s_{1} x_{1}+x_{j}\right)} .
\end{aligned}
$$

In the last line we have used the fact that $s_{1}^{2}=1$.

From expression (3.1) it is clear that $X$ can be written as a matrix product $X=D Y D$, where $D$ is a diagonal matrix with diagonal elements $1 /\left(s_{1} x_{1}+x_{i}\right)$ $(i>1)$ and where $Y$ is independent of $s_{1}$. It follows that the determinant of $Z_{N}$ is given by

$$
\operatorname{det} Z_{N}=\frac{g_{1}}{x_{1}} \operatorname{det}(D Y D)=\frac{g_{1}}{x_{1}} \operatorname{det}(Y) \operatorname{det}(D)^{2} .
$$

As the only factor that depends on the sign of $s_{1}$ appears to even power, and is therefore non-negative, we have proven that the $\operatorname{sign}$ of $\operatorname{det} Z_{N} \operatorname{does}$ not depend on the sign of $s_{1}$.

In a similar way, we can show that the sign of $\operatorname{det} Z_{N}$ does not depend on any of the signs of the $s_{i}$ 's. This ends the proof.

Proof of Theorem 2.2. It is a simple corollary of Proposition 2 that, under the conditions stated, the positive semidefiniteness of $Z_{N}$ for a given choice of signs of the $s_{i}$ 's implies positive semidefiniteness for any other choice. Indeed, according to Sylvester's criterion, a symmetric matrix is positive semidefinite if and only if all its principal minors are non-negative. In the case of $Z_{N}$, the principal $k \times k$ minors are determinants of the form $\operatorname{det} Z_{k}(k=1, \ldots, N)$, and according to Proposition 2. the signs of these determinants are independent of the signs of the $s_{i}$ 's appearing in them. 
Now consider, in particular, the case where $N$ is even, say $N=2 n$, and the $x_{i}$ are given by

$$
\left(x_{1}, \ldots, x_{n}, x_{n+1}, \ldots, x_{2 n}\right)=\left(y_{1}, \ldots, y_{n}, y_{1}+\epsilon, \ldots, y_{n}+\epsilon\right)
$$

for any positive $\epsilon$ small enough such that no two $x_{i}$ ever become equal when $\epsilon$ tends to 0 . Also let $g_{i}=g\left(x_{i}\right)$.

We will consider two choices for the $s_{i}$. Firstly, we set all $s_{i}=+1$. We then get the matrix

$$
K^{\prime}=\left(\begin{array}{cc}
K_{00} & K_{01} \\
K_{10} & K_{11}
\end{array}\right) .
$$

Secondly, with $s_{i}=+1$ for $i \leq n$ and $s_{i}=-1$ for $i>n$, we instead get

$$
K^{\prime \prime}=\left(\begin{array}{cc}
K_{00} & L_{01} \\
L_{10} & K_{11}
\end{array}\right) \text {. }
$$

As, according to Proposition 2, these matrices have the same signature (the same signs of the corresponding principal minors), this yields the equivalence of Theorem 2.2 .

It is now an easy matter to prove Theorem 2.1 .

Proof of Theorem 2.1. Let $N$ be a fixed integer, at least 2. By Proposition 1, if $g$ is an anti-Löwner function of order at least 2, then $g$ is continuous. Conversely, if the function $x \mapsto g(\sqrt{x}) \sqrt{x}$ is a non-negative matrix monotone function of order $N$, then surely $g$ must be continuous too. Thus, in any case, Theorem 2.2 applies to $g$.

Let $g$ be an anti-Löwner function of order $2 N$. Thus $K^{\prime}$ is positive. In the limit $\varepsilon \rightarrow 0$ we then find that $K_{g}+L_{g}$ and $K_{g}-L_{g}$ are positive, due to Theorem 2.2. A simple calculation shows that $K_{g}+L_{g}$ is equal to

$$
\begin{aligned}
K_{g}+L_{g} & =\left(\frac{g\left(x_{i}\right)+g\left(x_{j}\right)}{x_{i}+x_{j}}+\frac{g\left(x_{i}\right)-g\left(x_{j}\right)}{x_{i}-x_{j}}\right)_{i, j=1}^{N} \\
& =2\left(\frac{x_{i} g\left(x_{i}\right)-x_{j} g\left(x_{j}\right)}{x_{i}^{2}-x_{j}^{2}}\right)_{i, j=1}^{N},
\end{aligned}
$$

which is (up to an irrelevant factor of 2) the Löwner matrix of the function $x \mapsto$ $g(\sqrt{x}) \sqrt{x}$ in the points $x_{i}^{2}$. Hence, the function $x \mapsto g(\sqrt{x}) \sqrt{x}$ is a non-negative matrix monotone function of order $N$ on $\left(a^{2}, b^{2}\right)$.

In a similar way we find

$$
K_{g}-L_{g}=-2\left(x_{i} \frac{g\left(x_{i}\right) / x_{i}-g\left(x_{j}\right) / x_{j}}{x_{i}^{2}-x_{j}^{2}} x_{j}\right)_{i, j=1}^{N},
$$

which shows that the function $x \mapsto g(\sqrt{x}) / \sqrt{x}$ is a non-negative matrix monotone decreasing function of order $N$ on $\left(a^{2}, b^{2}\right)$.

\section{ACKNOWLEDGMENTS}

The author gratefully acknowledges the hospitality of the University of Ulm, Germany, and of the Institut Mittag-Leffler, Stockholm, where parts of this work have been done. Also many thanks to R. Bhatia and F. Hiai for comments on an earlier version of the manuscript. 


\section{REFERENCES}

1. R. Bhatia, Matrix Analysis, Springer, Heidelberg (1997). MR.1477662 (98i:15003)

2. R. Bhatia, Positive Definite Matrices, Princeton University Press, Princeton (2007). MR $2284176(2007 \mathrm{k}: 15005)$

3. W.F. Donoghue, Jr., Monotone Matrix Functions and Analytic Continuation, Springer, Heidelberg (1974). MR0486556 (58:6279)

4. M.K. Kwong, "On the definiteness of the solution of a certain matrix equation", Lin. Alg. Appl. 108, 177-198 (1988). MR959704 (89k:15022)

5. M.K. Kwong, "Some results on matrix monotone functions", Lin. Alg. Appl. 118, 129-153 (1989). MR995371(90e:47016)

Department of Mathematics, Royal Holloway, University of London, Egham TW20 0EX, United Kingdom

E-mail address: koenraad.audenaert@rhul.ac.uk 